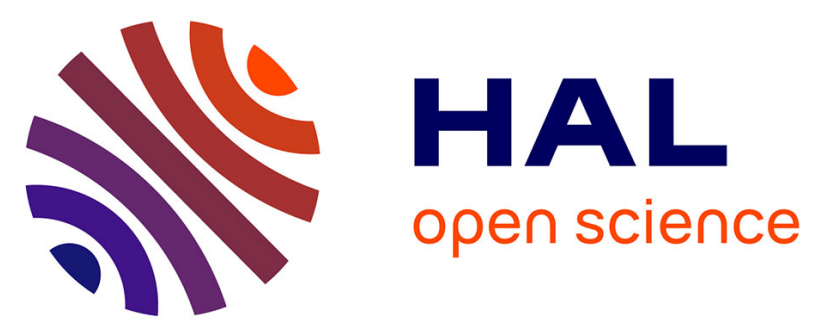

\title{
Influence of the internal geometry on the elastic properties of materials using 3D-printing of computer-generated random microstructures
}

Othmane Zerhouni, Maria-Gabriella Tarantino, Kostas Danas, Fei Hong

\section{- To cite this version:}

Othmane Zerhouni, Maria-Gabriella Tarantino, Kostas Danas, Fei Hong. Influence of the internal geometry on the elastic properties of materials using 3D-printing of computer-generated random microstructures. The Society of Exploration Geophysicists 2018 International Exposition and 88th Annual Meeting (SEG 2018), Oct 2018, Anaheim, CA, United States. 10.1190/segam2018-2998182.1. hal-01917370

HAL Id: hal-01917370

https://hal-polytechnique.archives-ouvertes.fr/hal-01917370

Submitted on 9 Nov 2018

HAL is a multi-disciplinary open access archive for the deposit and dissemination of scientific research documents, whether they are published or not. The documents may come from teaching and research institutions in France or abroad, or from public or private research centers.
L'archive ouverte pluridisciplinaire HAL, est destinée au dépôt et à la diffusion de documents scientifiques de niveau recherche, publiés ou non, émanant des établissements d'enseignement et de recherche français ou étrangers, des laboratoires publics ou privés. 


\section{Influence of the internal geometry on the elastic properties of materials using 3D-printing of computer-generated random microstructures Othmane Zerhouni*, Maria-Gabriella Tarantino and Kostas Danas, LMS Ecole Polytechnique and Fei Hong, TOTAL $R \& D$}

\section{SUMMARY}

Understanding elastic properties of rocks is a scientific challenge due to the complexity of their microstructures. This study combines the numerical tools to generate models for internal geometry of pores with the 3D printing technology in order to control the shape and size of pores as well as the distribution of pore's network inside the sample. Accuracy of the printing has been assessed by optical microscopy. The numerical and experimental tests conducted on generated microstructures show that the elastic properties are independent of the size of the pore. In turn, the shape of the pore has a strong effect on the elastic properties. This study shows that in case of multiple pore types, the ones with small aspect ratios have a strong impact though of minor volume fraction. The methodology that is developped can be extended to investigate the influence of more parameters in case of connected porosity and tests theories that have been proposed to link physical properties of reservoir rocks to their internal geometry.

\section{INTRODUCTION}

An important challenge in the developpement of reservoir models with high predictive capacity of production and the discovery of new wells is to understand and quantify the relationship between the internal structure of the rock and their elastic properties. The porous space of sedimentary rocks is characterized by heterogeneous and complex structures that spread over multiple scales. This multiscale geometry is difficult to capture with imaging techniques that are limited by the sample scales that can be observed and therefore need upscalling approach to get the overall properties of the field, i.e. need the determination of the equivalent permeability and bulk properties of each scale.

Laboratory analysis of rock properties, such as core flooding or mercury porosimetry, usually involves destructive testing which prevent from re-using the same rock in multiple tests. In turn, the numerical simulation offers a faster and nondestructive way to compute the elastic properties. However, the accurate characterization depends on the careful selection of the representative volume to overcome the dependence of the properties on the boundary conditions and the comparison between the experimental and computational testing does not involve the same scale of porosity due to the different size of samples that are used for each method.

The development of 3D printing manufacturing process has led to the development of new tools in geoscience to advance knowledge of the link between properties and microstructure. Such process has been used to derive the influence of the pro- cesses in sedimentary rock on the their experimental properties (Ishutov et al., 2017; Head and Vanorio, 2016). Their approach is based on image manipulation of the porous network of real rocks in order to highlight the microstructure resulting from a specific process such as compaction or dissolution. Their resulted images are then printed and tested in order to compare between experiments on printed samples and numerical simulations on images. The preliminary results shows good quantitative agreement for permeability related to the calculated porosity which shows the potential of additive manufacturing in developping methods to characterize the porous network influence on rock's properties and compare the experimental tests and the numerical simulation with the same samples. Another potential use of 3D printing consists of manufacturing the mathematical models of fractured rocks and porous network in order to validate the theoritical studies and the numerical methods (Suzuki et al., 2014). By controlling the fracture and pore geometry in the mathematical models which is in turn transferred into the experimental samples, this approach provide quantitative results of the influence of several aspects of the geometry such as the aperture of the interconnected porosity and the size of fractures on the flow properties.

The present study investigates the influence of the pore geometry on elastic properties of rocks. To that aim, we create random porous microstructures with controlled porosity and well-defined size and shape of pores. These microstructures are then experimentally and numerically tested to measure the linear elastic properties of the porous specimens. Using this methodology, we separately investigate the influence of each of the porosity, the size and the shape of voids on the elastic response of porous microstructures.

\section{MATERIAL AND METHOD}

We use a random sequential adsorption (RSA) algorithm (Rintoul and Torquato, 1997; Segurado and Llorca, 2002; LopezPamies et al., 2013) to construct the virtual microstructures of the present study. We specify to the algorithm the total porosity $c$ and parameters of each pore type $i$ which are its size $a_{i}$, its aspect ratio $\alpha_{i}$ and its volume fraction $c_{i}$ to create ellipsoidal voids randomly distributed in a cubic cell of size $L^{3}$. For illustration purposes, Fig. 1 shows three such RVEs comprising monodisperse spherical voids of increasing porosity $c=\{10,20,30\} \%$.

We build an experimental protocol to determine the elastic properties of the 3D-printed controlled microstructures. To that aim, we design virtual test specimens which ensure uniaxial stress conditions in the gage section by assembling lengthwise five representative cubic cells which are enclosed between the heads allowing us to mount the specimen onto the uni- 


\section{Influence of pore geometry on elastic properties}

a)

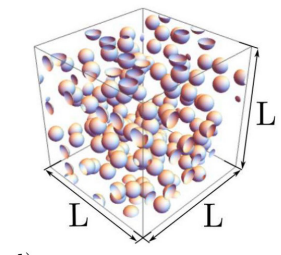

d)

e)

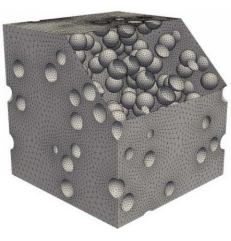

b)

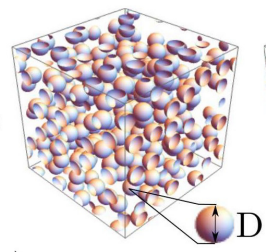

c)

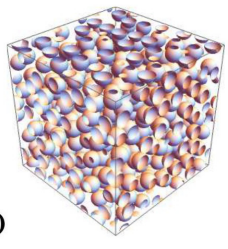

f)

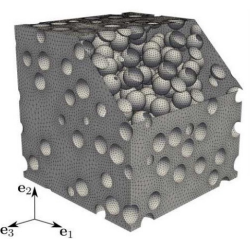

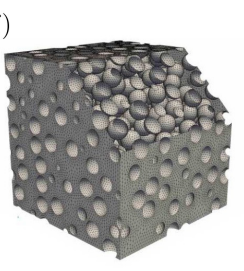

Figure 1: Representative cells of $12.8 \mathrm{~mm}$ (correponding to $0.5 \mathrm{in}$ ) with $N$ randomly distributed spherical particles of monodisperse sizes in a total porosity (a) $c=10 \%$ and $N=160$ (corresponding to $a=0.68 \mathrm{~mm}$ ), (b) $c=20 \%$ and $N=275$ (corresponding to $a=0.73 \mathrm{~mm}$ ), (c) $c=30 \%$ and $N=400$ (corresponding to $a=0.72$ ). (d-f) Representative meshes corresponding to the undeformed configuration of the representative cubic cells.

axial machine. These specimens are then 3D -printed using an acrylic photopolymer available in our EDEN 260VS 3Dprinter purchased by Stratasys. We determine the effective Young's modulus and Poisson's ratio of the 3D-printed random porous microstructures using uniaxial tensile loading while measing the axial and transverse strains by the extensometers as shown in Fig. 2. The elastic properties of the printing material are isotropic with Young modulus $E=1.4 \mathrm{GPa}$ and Poisson's ratio $v=0.42$ which correspond using equivalence between the P-wave velocity $V_{p}$ and $\mathrm{S}$-wave velocity $V_{s}$ with the static bulk modulus $\kappa$ and shear modulus $\mu$ in the case of isotropic material :

$$
V_{p}=\sqrt{\frac{\kappa+4 / 3 \mu}{\Phi}} \quad, \quad V_{s}=\sqrt{\frac{\mu}{\Phi}}
$$

The printing material wave velocities are around $V_{p}=1750 \mathrm{~m} . \mathrm{s}^{-1}$ and $V_{s}=660 \mathrm{~m} \cdot \mathrm{s}^{-1}$ and density $\Phi=1170 \mathrm{~kg} \cdot \mathrm{m}^{-3}$.

In turn, we use finite-element methods with a conforming quadratic mesh to obtain numerically the overall elastic properties of generated microstructures. Subsequently, the microstructures are discretized using the automatic mesh generator NETGEN (Schöberl, 1997) and simulated using ABAQUS software with periodic boundary conditions. For each set of parameters, we create four different samples to address the statistical deviation of the RSA process.

The host material is modeled by an isotropic linear elastic constitutive behavior with Young's Modulus and Poisson's ratio corresponding to those of the virgin matrix phase used during the printing.

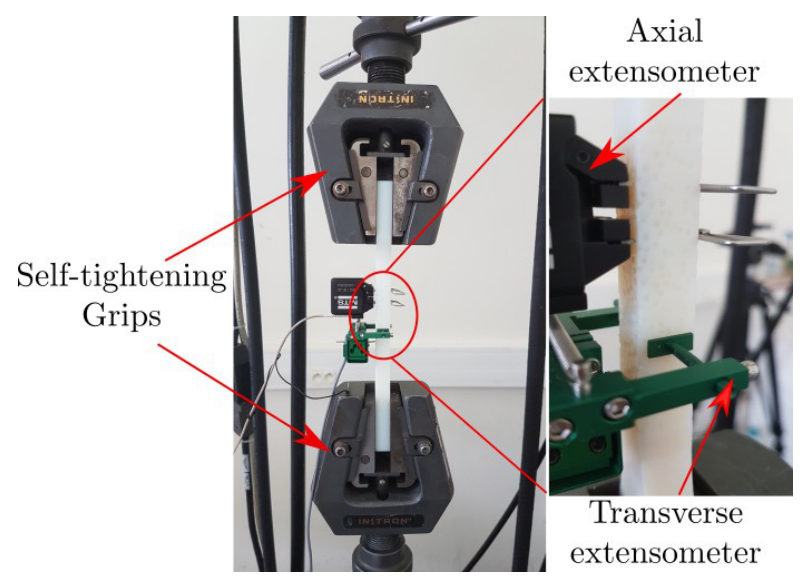

Figure 2: Experimental setup consisting of sample gripped by self-tightening grips mounted on a uni axial tensile machine which is connected to a LabView software to acquire the macroscopic measures of the axial and transverse strain.

\section{RESULTS AND DISCUSSION}

Effect of porosity on the elastic properties

We first focus our experimental and 3D printing efforts to isotropic random microstructures consisting of single sized (i.e. monodisperse) spherical voids embedded in a homogeneous solid matrix in order to study the effect on elastic properties only due of the variation of the total porosity.

We compare of their elastic effective properties with the HS isotropic bounds(Hashin and Shtrikman, 1963; Willis, 1977) given by

$\widetilde{\kappa}_{H S}=\frac{4(1-c) \mu_{m} \kappa_{m}}{4 \mu_{m}+3 c \kappa_{m}} \quad, \quad \widetilde{\mu}_{H S}=\frac{(1-c)\left(8 \mu_{m}+9 \kappa_{m}\right) \mu_{m}}{4 \mu_{m}(2+3 c)+3 \kappa_{m}(3+2 c)}$

where $\kappa_{m}$ and $\mu_{m}$ denote the bulk and shear moduli of the matrix phase, respectively and $c$ the volume fraction of the inclusion phase or porosity in the present context.

Results in Figs $3 \mathrm{a}$ and $3 \mathrm{~b}$ show that increasing the porosity creates larger void regions which implies significant interactions between voids and softens the material.

\section{Pore size effect}

Pore size is pointed out as an important parameter in the variation of $\mathrm{P}$-wave and S-wave velocities during pore-type characterization that links between microscopic images from thin section and experimental tests over the core sample. However, none of the existing analytical estimates take into account the size of the pore in their predictions but only the shape of pore and the porosity. In order to determine if pore size affects the elastic properties, we generate microstructures that have the same porosity $c=\{5 \%, 10 \%, 15 \%, 20 \%, 25 \%\}$ and spherical pores of decreasing size. Figure 4 shows different realizations of these microstructures for $c=20 \%$ with pore size being the only changing parameter. FE results in Fig 5 are very similar for all computed sizes showing no effect of size on the elastic properties of the material.The variations that can be observed are due to statistical sampling. 


\section{Influence of pore geometry on elastic properties}

\section{Pore shape effect}

a)
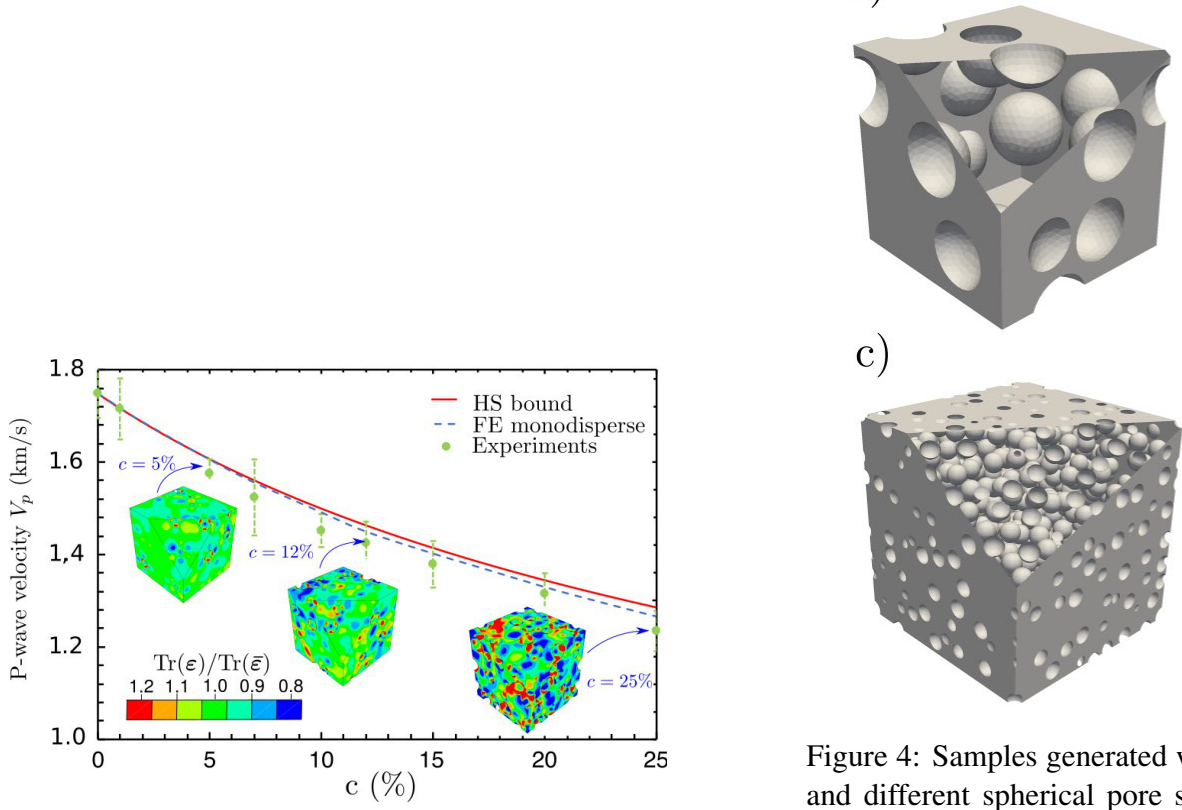

(a)

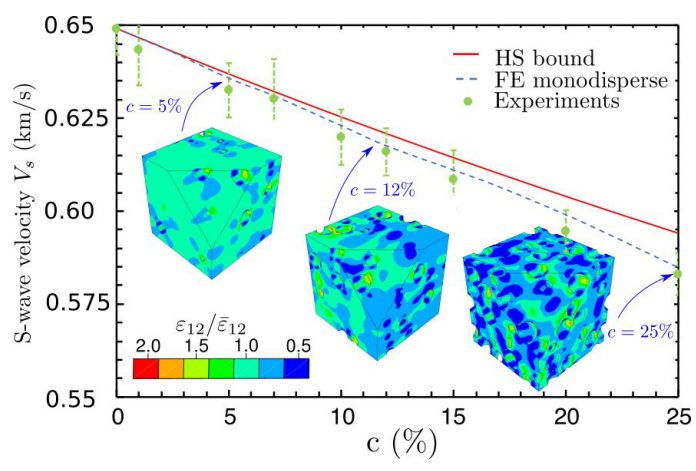

(b)

Figure 3: Effective (a) P-wave velocity $V_{p}$, (b) S-wave velocity $V_{s}$ of microstructures with spherical single-size voids as a function of the porosity $c$. Comparison between the analytical HS bounds, FE monodisperse numerical estimates and experimental results.
C)

b)

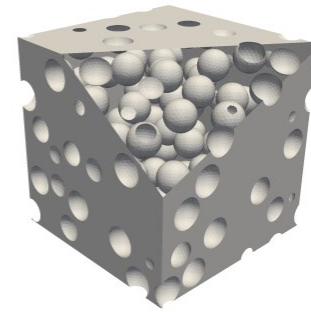

d)

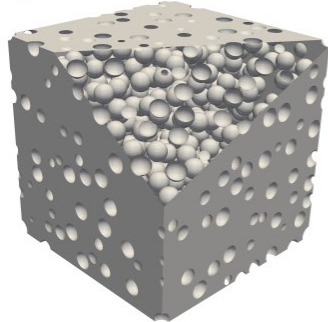

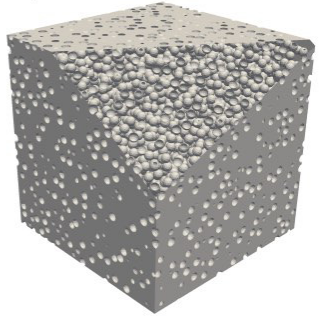

Figure 4: Samples generated with the same porosity $c=20 \%$ and different spherical pore size in a cell of $12.8 \mathrm{~mm}$ (correponding to $0.5 \mathrm{in}$ ) with: a) radius $a=2.15 \mathrm{~mm}$, b) radius $a=1 \mathrm{~mm}, \mathrm{c}$ ) radius $a=0.55 \mathrm{~mm}, \mathrm{~d}$ ) radius $a=0.27 \mathrm{~mm}$

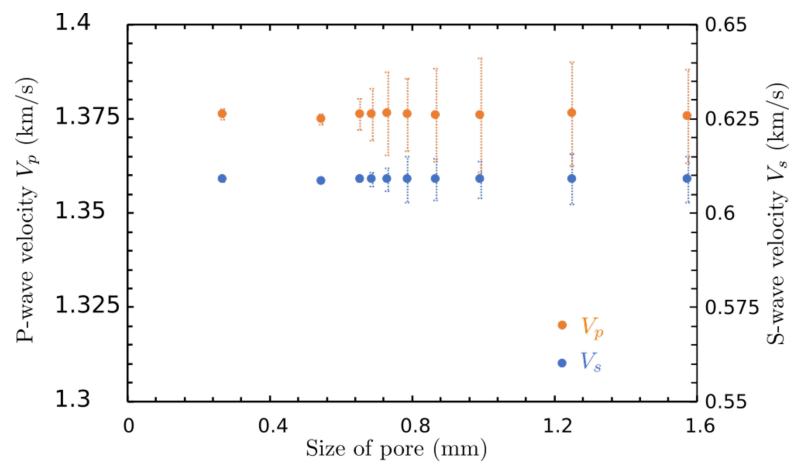

Figure 5: effective P-wave and S-wave velocities obtained numerically using FEM for various size of pore in cubic sample of $L=12 \mathrm{~mm}$ with fixed porosity $c=20 \%$

In order to link between the pore-type distribution and the elastic properties of a porous material, one approach by Weger et al. (2009); Fournier et al. (2011) consists in deriving two parameters $\left(\alpha_{\kappa}\right.$ and $\alpha_{\mu}$ ) corresponding to the aspect ratio of ellispodal pores in the different effective medium (DEM) model (Berryman, 1992; Mavko et al., 2009) that has similar P-wave and $\mathrm{S}$-wave velocities to the well-log acoustic response of the microstructure. The accuracy of pore-type classification based on such approach is closely dependent on understanding the most influent pore type among the pore types withdrawn, from thin section observations. In order to quantify the uncertainties related to this choice, we generated microstructures with controlled porosity $c \in[1 \%, 25 \%]$. 


\section{Influence of pore geometry on elastic properties}

We use aspect ratios taken from petroacoustic parameter $\alpha_{\kappa}$ obtained by studying carbonates rocks (Fournier et al., 2017) to generate microstructures with different pore shapes and volume fraction of each. Realizations of such microstructures are shown in Fig. 6 to highlight the different internal geometry that can be found for the same porosity $c=20 \%$. We use numeri-
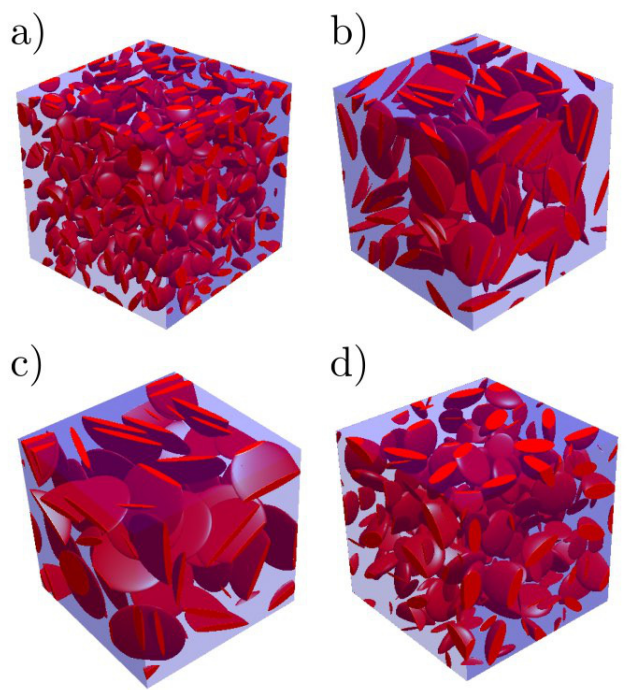

Figure 6: Microstructures with porosity $c=20 \%$ composed of pores of different aspect ratios (AR) : a) $100 \%$ of ellipsoidal pores of $\mathrm{AR}=0.3, \mathrm{~b}) 100 \%$ of ellipsoidal pores of $\mathrm{AR}=0.13$, c) $100 \%$ of ellipsoidal pores of $\mathrm{AR}=0.1, \mathrm{~d}) 90 \%$ of ellipsoidal pores of $\mathrm{AR}=0.3$ and $10 \%$ of ellipsoidal pores of $\mathrm{AR}=0.1$

cal simulation to compute the effective properties of each microstructures. Figures $7 \mathrm{a}$ and $7 \mathrm{~b}$ highlights the effect of pore shape on the elastic properties of the material. However, the results shown are assuming isotropic effective behavior to retrieve bulk and shear modulus and subsequently $\mathrm{P}$-wave and $\mathrm{S}$ wave velocities. This assumption is far from being consisting with the elastic properties of the microstructures that exhibits high anisotropy in case of small AR.

To highlight the importance of this study, we compute the deviation from the effective result if considering that the most present pore shape is the most influencing on the elastic properties. Results in Fig. 8 show that small aspect ratio are more impacting the elastic properties and that the acoustic signature of the microstructure is highly related to the presence of these highly elongated pores.

\section{ACKNOWLEDGMENTS}

Authors acknowledge support from TOTAL S.A. The computational part of this work was also supported by the ANR under contract number ANR-10-EQPX-37.
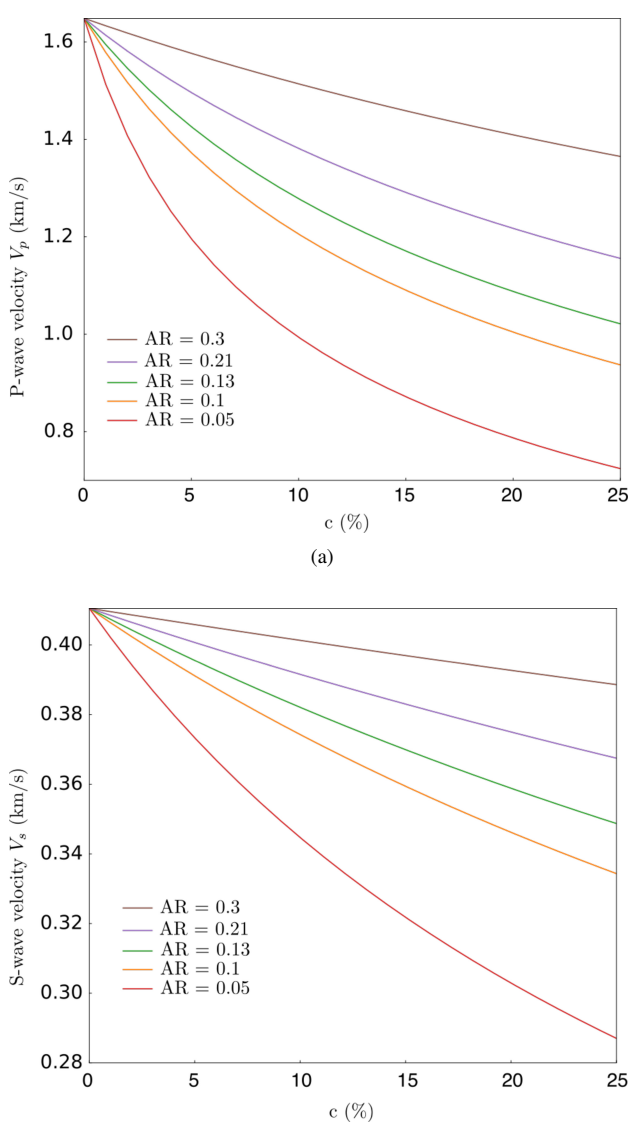

(b)

Figure 7: Results of :a) P-wave velocity b) S-wave velocity, variations due to change in the aspect ratio of the ellipsoidal voids.

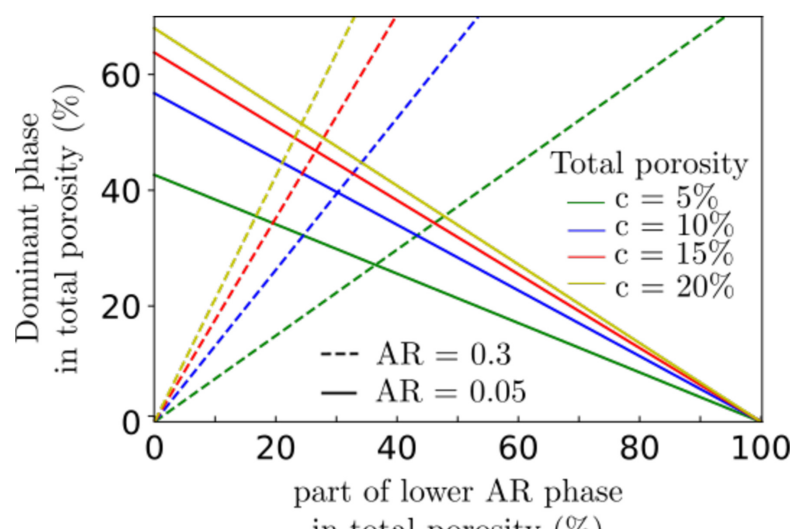

in total porosity (\%)

Figure 8: Dominant phase Error in the elastic properties when considering a phase dominant over another in two pore type coexisting in the same microstructure. 


\section{Influence of pore geometry on elastic properties}

\section{REFERENCES}

Berryman, J. G., 1992, Single-scattering approximations for coefficients in biot's equations of poroelasticity: The Journal of the Acoustical Society of America, 91, 551-571.

Fournier, F., P. Lonide, K. Biscarrat, A. Gallois, J. Borgomano, and A. Foubert, 2011, Elastic properties of microporous cemented grainstones: 76, 175-.

Fournier, F., M. Pellerin, Q. Villeneuve, T. Teillet, F. Hong, E. Poli, J. Borgomano, P. Lonide, and A. Hairabian, 2017, The equivalent pore aspect ratio as a tool for pore type prediction in carbonate reservoirs: AAPG Bulletin.

Hashin, Z., and S. Shtrikman, 1963, A variational approach to the theory of the elastic behaviour of multiphase materials: Journal of the Mechanics and Physics of Solids, 127-140.

Head, D., and T. Vanorio, 2016, Effects of changes in rock microstructures on permeability: 3 -d printing investigation: Geophysical Research Letters, 43, 7494-7502. (2016GL069334).

Ishutov, S., F. J. Hasiuk, D. Jobe, and S. Agar, 2017, Using resin-based $3 \mathrm{~d}$ printing to build geometrically accurate proxies of porous sedimentary rocks: Groundwater.

Lopez-Pamies, O., T. Goudarzi, and K. Danas, 2013, The nonlinear elastic response of suspensions of rigid inclusions in rubber: Ii-a simple explicit approximation for finiteconcentration suspensions: Journal of the Mechanics and Physics of Solids, 61, $19-37$.

Mavko, G., T. Mukerji, and J. Dvorkin, 2009, The rock physics handbook: Tools for seismic analysis of porous media, 2nd ed.: Cambridge University Press.

Rintoul, M., and S. Torquato, 1997, Reconstruction of the structure of dispersions: Journal of Colloid and Interface Science, 186, 467 - 476.

Schöberl, J., 1997, Netgen an advancing front 2d/3d-mesh generator based on abstract rules: Computing and Visualization in Science, 1, 41-52.

Segurado, J., and J. Llorca, 2002, A numerical approximation to the elastic properties of sphere-reinforced composites: Journal of the Mechanics and Physics of Solids, $\mathbf{5 0 .}$

Suzuki, A., K. Li, and R. Horne, 2014, fracture network, flow experiment, 3d printer, ct scan images: Stanford Geothermal Workshop, n/a-n/a.

Weger, R., G. Eberli, G. Baechle, J. Massaferro, and Y. Sun, 2009, Quantification of pore structure and its effect on sonic velocity and permeability in carbonates: 10, 1297-1317.

Willis, J., 1977, Bounds and self-consistent estimates for the overall properties of anisotropic composites: Journal of the Mechanics and Physics of Solids, 25, 185 - 202. 